\title{
Symbol Error Performance Analysis and Comparison between Alamouti and MRRC Diversity Combining Scheme for Wireless Communication
}

\author{
A. Khatoon ${ }^{1}$ and M. S. Rahman ${ }^{2}$ \\ ${ }^{1}$ Faculty of EECE, Military Institute of Science and Technology, Dhaka-1216, Bangladesh \\ ${ }^{2}$ Transmission Engineer, N\&TC, LM Ericsson Bangladesh Ltd., Bangladesh \\ Received 6 August 2009, accepted in final revised form 13 December 2009
}

\begin{abstract}
The symbol error performance of a wireless communication system is analyzed using diversity combining scheme in Rayleigh fading channels. The performance of the system is compared using Alamouti scheme and maximal-ratio receiver combining (MRRC) scheme. The performance results of the system are evaluated by numerical computation and by simulation using MATLAB. A detailed analysis and exact (closed-form) expressions of the probability of error of the wireless communication systems in Rayleigh fading channels are provided for both M-ary phase-shift keying (M-PSK) and M-ary quadrature amplitude modulation (M-QAM) schemes. Monte Carlo simulation shows exact match with the theoretical results.
\end{abstract}

Keywords: Alamouti scheme; M-PSK; M-QAM; MRRC; Symbol error probability (SEP).

(c) 2010 JSR Publications. ISSN: 2070-0237 (Print); 2070-0245 (Online). All rights reserved.

DOI: $10.3329 /$ jsr.v2i1.3000

J. Sci. Res. 2 (1), 54-66 (2010)

\section{Introduction}

Diversity techniques have almost exclusively been applied to base stations to improve their reception quality. This is because it is more economical to add equipment to base stations rather than the remote units. For this reason, transmit diversity schemes are very attractive. Receiver diversity is a form of space diversity, where there are multiple antennas at the receiver. Numerous research works have been carried out during the last few years on the performance evaluation of wireless communication system using diversity technique. Wireless Communications and Networking including coding, different multiplexing and multiple accesses, forms of diversity, generations of communications, WiMAX and many topics related to wireless communications are well discussed [1-4]. Discussions on diversity technique, multiple accesses and various

\footnotetext{
${ }^{1}$ Corresponding author: afsana_shimu@yahoo.com
} 
information on wireless communication system can also be found in the Wikipedia [5]. A simple two-branch transmit diversity scheme is presented in ref. [6]. Using two transmit antennas and one receive antenna the scheme provides the same diversity order as maximal-ratio receiver combining (MRRC) with one transmit antenna, and two receive antennas. It is also shown that the scheme may easily be generalized to two transmit antennas and $\mathrm{M}$ receive antennas to provide a diversity order of 2M. Based on Alamouti's work, Tarokh et al. [7] proposed orthogonal space-time block codes (OSTBCs) combining the orthogonal coding method and the simple diversity technique. Tarokh [8] studies the encoding and decoding algorithms for various codes and provides simulation results demonstrating their performance. A squaring method to simplify the decoding of orthogonal space-time block codes in a wire-less system with an arbitrary number of transmit and receive antennas is reported in ref. [9].

In this paper, we present the theoretical analysis to evaluate the symbol error performance of a wireless communication system using Alamouti-scheme and MRRC diversity combining scheme. Closed-form expressions of signal-to-noise-ratio (SNR) are analytically derived for both Alamouti scheme and MRRC scheme. Using these SNR equations Exact expressions of symbol error probability (SEP)are derived analytically for up to $2 \mathrm{Tx}-2 \mathrm{Rx}$ transmit diversity scheme and as well as 4 branch receiver diversity scheme for binary phase-shift keying (BPSK), quadrature phase shift-keying( QPSK) and M-ary quadrature amplitude modulation (M-QAM) schemes. The symbol error performance results are numerically evaluated and simulated using MATLAB software.

\section{Transmit Diversity Scheme}

\subsection{Two-branch transmit diversity with one receiver}

The combiner builds the following combined signal that is sent to the maximum likelihood detector. We can write as [6]

$$
\hat{x}_{0}=\left[\left|h_{0}\right|^{2}+\left|h_{1}\right|^{2}\right] x_{0}+h_{0}^{*} n_{0}+h_{1} n_{1}^{*}
$$

The signal power

$$
P_{x}=\left[\left|h_{0}\right|^{2}+\left|h_{1}\right|^{2}\right]^{2} E\left\{|x|^{2}\right\}
$$

Assume $\frac{E_{s}}{n_{T}}=\frac{1}{2} E\left\{\left|x_{1}\right|^{2}\right\}=\cdots=\frac{1}{2} E\left\{\left|x_{B}\right|^{2}\right\}=\frac{\sigma_{x}^{2}}{2}$, where $E\{$.$\} denotes expectation, \sigma_{x}^{2}$ is the average symbol power and $n_{T}$ means number of transmitter. The signal-to-noise-ratio (SNR) becomes

$$
S N R=\frac{P_{X}}{P_{W}}=\frac{\left[\left|h_{0}\right|^{2}+\left|h_{1}\right|^{2}\right] \sigma_{x}^{2}}{2 \sigma_{w}^{2}}
$$

where, $\sigma_{w}^{2}$ is the noise power. 


\subsection{Two-branch transmit diversity with two receiver}

The received signal can then be expressed as [6]

$$
\begin{aligned}
& r_{0}=h_{0} x_{0}+h_{1} x_{1}+n_{0} \\
& r_{1}=-h_{0} x_{1}^{*}+h_{1} x_{0}^{*}+n_{1} \\
& r_{2}=h_{2} x_{0}+h_{3} x_{1}+n_{2} \\
& r_{3}=-h_{2} x_{1}^{*}+h_{3} x_{0}^{*}+n_{3}
\end{aligned}
$$

where $n_{0}, n_{1}, n_{2}$ and $n_{3}$ are complex random variables representing receiver thermal noise and interference. The combiner builds the following signal that is sent to the maximum likelihood detector:

$$
\hat{x}_{0}=h_{0}^{*} r_{0}+h_{1} r_{1}^{*}+h_{2}^{*} r_{2}+h_{3} r_{3}^{*}
$$

Substitution of Eq. (4) in Eq. (5) gives

$$
\hat{x}_{0}=\left[\left|h_{0}\right|^{2}+\left|h_{1}\right|^{2}+\left|h_{2}\right|^{2}+\left|h_{3}\right|^{2}\right] x_{0}+h_{0}^{*} n_{0}+h_{1} n_{1}^{*}+h_{2}^{*} n_{2}+h_{3} n_{3}^{*}
$$

The signal-to-noise-ratio (SNR) becomes

$$
S N R=\frac{P_{X}}{P_{W}}=\frac{\left[\left|h_{0}\right|^{2}+\left|h_{1}\right|^{2}+\left|h_{2}\right|^{2}+\left|h_{3}\right|^{2}\right] \sigma_{x}^{2}}{2 \sigma_{w}^{2}}
$$

\section{Receiver Diversity: MRRC Scheme}

\subsection{One transmitter - two receivers}

\section{Two branch maximal-ratio receive combining (MRRC) scheme}

The receiver combining scheme for two-branch MRRC is as follows:

$$
\hat{x}_{0}=h_{0}^{*} r_{0}+h_{1}^{*} r_{1}=\left|h_{0}\right|^{2}+\left|h_{1}\right|^{2}+h_{0}^{*} n_{0}+h_{1}^{*} n_{1}
$$

The signal-to-noise-ratio (SNR) becomes

$$
S N R=\frac{P_{X}}{P_{W}}=\frac{\left[\left|h_{0}\right|^{2}+\left|h_{1}\right|^{2}\right] \sigma_{x}^{2}}{\sigma_{w}^{2}}
$$

\subsection{One transmitter - four receivers}

\section{Four branch maximal-ratio receive combining (MRRC) scheme}

The resulting received baseband signals are 
$r_{1}=h x_{1}+n_{1}$

The receiver combining scheme for the four-branch MRRC is as follows:

$$
\hat{x}_{0}=h_{0}^{*} r_{0}+h_{1}^{*} r_{1}=\left|h_{0}\right|^{2}+\left|h_{1}\right|^{2}+\left|h_{2}\right|^{2}+\left|h_{3}\right|^{2}+h_{0}^{*} n_{0}+h_{1}^{*} n_{1}+h_{2}^{*} n_{2}+h_{3}^{*} n_{3}
$$

The signal-to-noise-ratio (SNR) becomes

$$
S N R=\frac{P_{X}}{P_{W}}=\frac{\left[\left|h_{0}\right|^{2}+\left|h_{1}\right|^{2}+\left|h_{2}\right|^{2}+\left|h_{3}\right|^{2}\right] \sigma_{x}^{2}}{\sigma_{w}^{2}}
$$

\section{Probability of Error Analysis}

\subsection{One transmitter - one receiver}

From (3) the instantaneous SNR per symbol $\eta$ for single input single output (SISO) [10] case becomes,

$$
\eta=\Delta|h|^{2}, \quad \text { where } \Delta=\frac{\sigma_{x}^{2}}{\sigma_{w}^{2}}
$$

Let $v=|h|^{2}$ which has a central chi-square distribution with $2 n_{T} n_{R}$ freedom. So, the PDF [11] of $\mathrm{V}$ becomes $p_{v}(v)=e^{-v}$. The PDF of $\eta$ becomes

$$
P_{\eta}(\eta)=\frac{1}{\Delta} \exp \left(\frac{-\eta}{\Delta}\right)
$$

Let $P_{e}(\eta)$ be the bit or symbol error probability for a given modulation scheme(MPSK or M-QAM)at the instantaneous SNR value $\eta$.Then the average probability of error $P_{e}^{\text {SISO }}$ for the SISO system can be calculate as

$$
P_{e}^{S I S O}=\int_{0}^{\infty} P_{e}(\eta) p_{\eta}(\eta) d \eta
$$

\subsubsection{M-ary PSK modulation}

The symbol- error probability $P_{e}(\eta)$ for M-ary PSK modulation can be given as [12]

$$
P_{e}(\eta)=\frac{1}{\pi} \int_{0}^{\beta} \exp \left(\frac{-\eta a}{\sin ^{2} \theta}\right) d \theta
$$

where, $a=\sin ^{2}(\pi / M)$ and $\beta=\pi-\pi / M$. Substitution of Eq. (13) and Eq. (15) in (14) gives 


$$
P_{e}^{S I S O}=\frac{1}{\pi \Delta} \int_{0}^{\beta} \int_{0}^{\infty} \exp \left[-\left(\frac{a}{\sin ^{2} \theta}+\frac{1}{\Delta}\right) \eta\right] d \eta d \theta \quad=\frac{\beta}{\pi}-\frac{1}{\pi} \sqrt{\frac{b_{1}}{b_{1}+1}} \tan ^{-1}\left\{\sqrt{\frac{b_{1}+1}{b_{1}} \tan \beta}\right\}
$$

where, $b_{1}=\Delta a=\Delta \sin ^{2}(\pi / M)$

\subsection{2. $M-Q A M$ modulation}

The symbol-error probability $P_{e}(\eta)$ for square M-QAM modulation can be given as [12]

$$
P_{e}(\eta)=\frac{4 q}{\pi} \int_{0}^{\pi / 2} \exp \left(\frac{-\eta g}{\sin ^{2} \theta}\right) d \theta-\frac{4 q^{2}}{\pi} \int_{0}^{\pi / 4} \exp \left(\frac{-\eta g}{\sin ^{2} \theta}\right) d \theta
$$

where, $q=1-1 / \sqrt{M}$ and $g=\frac{3}{2}(M-1)$. The average symbol-error probability $P_{e}^{\text {SISO }}$ can be determined by evaluating the integral in (14), where $p_{\eta}(\eta)$ is given by (15).Lets define the function $\psi_{1}(\Delta, a, \beta)$ as

$$
\left.\psi_{1}(\Delta a, \beta)=\frac{1}{\pi \Delta} \int_{0}^{\beta \infty} \int_{0}^{\infty} \exp \left[-\left(\frac{a}{\sin ^{2} \theta}+\frac{1}{\Delta}\right)\right]\right] d \eta d \theta
$$

$\psi_{1}(\Delta, a, \beta)$ is given by the right-hand-side expression in (16), where $b_{1}=\Delta a$.Using $\psi_{1}(\Delta, a, \beta)$ the average symbol-error probability for M-QAM modulation can be given as

$$
P_{e}^{S I S O}=4 q \psi_{1}(\Delta, g, \pi / 2)-4 q^{2} \psi_{1}(\Delta, g, \pi / 4)
$$

where,

$$
\begin{aligned}
& \psi_{1}=(\Delta, g, \pi / 2)=\frac{1}{2}\left(1-\sqrt{\frac{c}{c+1}}\right) \\
& \psi_{1}(\Delta, g, \pi / 4)=\frac{1}{4}-\frac{1}{\pi} \sqrt{\frac{c}{c+1}} \tan ^{-1}\left(\sqrt{\frac{c+1}{c}}\right)
\end{aligned}
$$

and $c=g \Delta$.

\subsection{Two transmitters and one receiver}

From (3) the instantaneous SNR per symbol $\lambda$ becomes,

$$
\begin{aligned}
& \lambda=\frac{\Delta}{2}\left[\left|h_{0}\right|^{2}+\left|h_{1}\right|^{2}\right], \text { where } \Delta=\frac{\sigma_{x}^{2}}{\sigma_{w}^{2}} \text {. The PDF of } \lambda \text { becomes } \\
& P_{\lambda}(\lambda)=\frac{4 \lambda}{\Delta^{2}} \exp \left(\frac{-2 \lambda}{\Delta}\right)
\end{aligned}
$$

The average probability of error $P_{e}^{2 T_{x}-1 R_{x}}$ for the system can be calculated as 


$$
P_{e}^{2 T_{x}-1 R_{x}}=\int_{0}^{\infty} P_{e}(\lambda) p_{\lambda}(\lambda) d \lambda
$$

\subsubsection{M-ary PSK modulation}

The symbol-error probability $P_{e}(\lambda)$ for M-ary PSK modulation can be given as

$$
P_{e}(\lambda)=\frac{1}{\pi} \int_{0}^{\beta} \exp \left(\frac{-\lambda a}{\sin ^{2} \theta}\right) d \theta
$$

where, $a=\sin ^{2}(\pi / M)$ and $\beta=\pi-\pi / M$. Substitution of (22) and (24) in (23) and by performing the integral the equation becomes

$$
P_{e}^{2 T_{x}-1 R_{x}}=\frac{\beta}{\pi}+\frac{1}{\pi\left(b_{1}+2\right)}\left[\frac{b_{1} \sin \beta \cos \beta}{\left(b_{1}+2 \sin ^{2} \beta\right)}-\left(b_{1}+3\right) \sqrt{\frac{b_{1}}{b_{1}+2}} \tan ^{-1}\left\{\sqrt{\frac{b_{1}+2}{b_{1}}} \tan \beta\right\}\right]
$$

\subsubsection{M-QAM modulation}

The symbol-error probability $P_{e}(\lambda)$ for square M-QAM modulation can be given as

$$
P_{e}(\lambda)=\frac{4 q}{\pi} \int_{0}^{\pi / 2} \exp \left(\frac{-\lambda g}{\sin ^{2} \theta}\right) d \theta-\frac{4 q^{2}}{\pi} \int_{0}^{\pi / 4} \exp \left(\frac{-\lambda g}{\sin ^{2} \theta}\right) d \theta
$$

Let us define the function $\psi_{2}(\Delta, a, \beta)$ as

$$
\psi_{2}(\Delta, a, \beta)=\frac{1}{\pi \Delta^{2}} \int_{0}^{\beta} \int_{0}^{\infty} \exp \left[-\left(\frac{a}{\sin ^{2} \theta}+\frac{1}{\Delta}\right) \lambda\right] d \lambda d \theta
$$

$\psi_{2}(\Delta, a, \beta)$ is given by the right-hand-side expression in (25). The average symbolerror probability for M-QAM modulation can be given as

$$
P_{e}^{2 T_{x}-1 R_{x}}=4 q \psi_{2}(\Delta, g, \pi / 2)-4 q^{2} \psi_{2}(\Delta, g, \pi / 4)
$$

where,

$$
\begin{aligned}
& \psi_{2}=(\Delta, g, \pi / 2)=\frac{1}{2}\left(1-\frac{c+3}{c+2} \sqrt{\frac{c}{c+2}}\right) \\
& \psi_{2}(\Delta, g, \pi / 4)=\frac{1}{4}+\frac{1}{\pi(c+2)}\left\{\frac{c}{2(c+1)}-(c+3) \sqrt{\frac{c}{c+1}} \tan ^{-1}\left(\sqrt{\frac{c+2}{c}}\right)\right\}
\end{aligned}
$$

and $c=g \Delta$.

\subsection{One transmitter and two receivers}

The PDF of instantaneous SNR per symbol $\lambda_{1}$ becomes

$$
P_{\lambda 1}\left(\lambda_{1}\right)=\frac{\lambda_{1}}{\Delta^{2}} \exp \left(\frac{-\lambda_{1}}{\Delta}\right)
$$

The average probability of error $P_{e}^{1 T_{x}-2 R_{x}}$ for the system can be calculated as 


$$
P_{e}^{1 T_{x}-2 R_{x}}=\int_{0}^{\infty} P_{e}\left(\lambda_{1}\right) p_{\lambda_{1}}\left(\lambda_{1}\right) d \lambda_{1}
$$

\subsubsection{M-ary PSK modulation}

The symbol-error probability $P_{e}\left(\lambda_{1}\right)$ for M-ary PSK modulation can be given as

$$
P_{e}\left(\lambda_{1}\right)=\frac{1}{\pi} \int_{0}^{\beta} \exp \left(\frac{-\lambda_{1} a}{\sin ^{2} \theta}\right) d \theta
$$

Substitution of (30) and (32) in (31) gives and by performing the integral the equation becomes

$$
P_{e}^{1 T_{x}-2 R_{x}}=\frac{\beta}{\pi}+\frac{1}{2 \pi\left(b_{1}+1\right)}\left[\frac{b_{1} \sin \beta \cos \beta}{\left(b_{1}+\sin ^{2} \beta\right)}-\left(2 b_{1}+3\right) \sqrt{\frac{b_{1}}{b_{1}+1}} \tan ^{-1}\left\{\sqrt{\frac{b_{1}+1}{b_{1}}} \tan \beta\right\}\right]
$$

\subsubsection{M-QAM modulation}

The symbol-error probability $P_{e}\left(\lambda_{1}\right)$ for square M-QAM modulation can be given as

$$
P_{e}\left(\lambda_{1}\right)=\frac{4 q}{\pi} \int_{0}^{\pi / 2} \exp \left(\frac{-\lambda_{1} g}{\sin ^{2} \theta}\right) d \theta-\frac{4 q^{2}}{\pi} \int_{0}^{\pi / 4} \exp \left(\frac{-\lambda_{1} g}{\sin ^{2} \theta}\right) d \theta
$$

$\psi_{2}(\Delta, a, \beta)$ is given by the right-hand-side expression in Eq. (33). The average symbol-error probability for M-QAM modulation can be given as

$$
P_{e}^{2 T_{x}-1 R_{x}}=4 q \psi_{2}(\Delta, g, \pi / 2)-4 q^{2} \psi_{2}(\Delta, g, \pi / 4)
$$

where,

$$
\begin{aligned}
& \psi_{2}=(\Delta, g, \pi / 2)=\frac{1}{2}\left(1-\frac{(2 c+3)}{2(c+1)} \sqrt{\frac{c}{c+1}}\right) \\
& \psi_{2}(\Delta, g, \pi / 4)=\frac{1}{4}+\frac{1}{8(c+1)}\left\{\frac{c}{(2 c+1)}-(2 c+3) \sqrt{\frac{c}{c+1}} \tan ^{-1}\left(\sqrt{\frac{c+1}{c}}\right)\right\}
\end{aligned}
$$

\subsection{One transmitter - four receivers}

From (12) the instantaneous SNR per symbol $\varepsilon_{1}$ becomes, $\varepsilon_{1}=\Delta\left[\left|h_{0}\right|^{2}+\left|h_{1}\right|^{2}+\left|h_{2}\right|^{2}+\left|h_{3}\right|^{2}\right], \quad$ where $\Delta=\frac{\sigma_{x}^{2}}{\sigma_{w}^{2}}$. The PDF of $\varepsilon_{1}$ becomes

$$
P_{\varepsilon}\left(\varepsilon_{1}\right)=\frac{\varepsilon_{1}^{3}}{6 \Delta^{4}} \exp \left(\frac{-\varepsilon_{1}}{\Delta}\right)
$$

The average probability of error $P_{e}^{1 T_{x}-4 R_{x}}$ for the system can be calculated as 


$$
P_{e}^{4 T_{x}-1 R_{x}}=\int_{0}^{\infty} P_{e}\left(\varepsilon_{1}\right) p_{\varepsilon}\left(\varepsilon_{1}\right) d \varepsilon_{1}
$$

\subsubsection{M-ary PSK modulation}

The symbol-error probability $P_{e}\left(\varepsilon_{1}\right)$ for M-ary PSK modulation can be written as

$$
P_{e}\left(\varepsilon_{1}\right)=\frac{1}{\pi} \int_{0}^{\beta} \exp \left(\frac{-\varepsilon_{1} a}{\sin ^{2} \theta}\right) d \theta
$$

Substitution of (37) and (39) in (38) and by performing the integral [13] the equation becomes

$$
P_{e}^{4 T_{x}-1 R_{x}}=\frac{1}{\pi}\left[\beta+\frac{1}{\left(b_{1}+1\right)^{3}}\left[\begin{array}{l}
\left\{\frac{\left(\frac{29}{16} b_{1}+\frac{13}{8} b_{1}^{2}+\frac{1}{2} b^{3}\right) \sin \theta \cos \theta}{\left(b_{1}+\sin ^{2} \theta\right)}\right\}+\frac{b_{1}^{3} \tan \theta \cos ^{6} \theta}{6\left(b_{1}+\sin ^{2} \theta\right)^{3}} \\
+\left\{\frac{\left(-\frac{19}{24} b_{1}^{2}-\frac{1}{4} b_{1}^{3}\right) \tan \theta \cos ^{4} \theta}{\left(b_{1}+\sin ^{2} \theta\right)^{2}}\right\}+\left(-\frac{35 b_{1}}{16}-\frac{35 b_{1}^{2}}{8}-\frac{7}{2} b_{1}^{3}-b_{1}^{4}\right) \\
\left\{\frac{1}{\sqrt{b_{1}\left(b_{1}+1\right)}} \tan ^{-1}\left(\sqrt{\frac{b_{1}+1}{b_{1}}} \tan \theta\right)\right\}
\end{array}\right]\right.
$$

\subsection{2. $M-Q A M$ modulation}

The symbol-error probability $P_{e}\left(\varepsilon_{1}\right)$ for square M-QAM modulation can be given as

$$
P_{e}\left(\varepsilon_{1}\right)=\frac{4 q}{\pi} \int_{0}^{\pi / 2} \exp \left(\frac{-\varepsilon_{1} g}{\sin ^{2} \theta}\right) d \theta-\frac{4 q^{2}}{\pi} \int_{0}^{\pi / 4} \exp \left(\frac{-\varepsilon_{1} g}{\sin ^{2} \theta}\right) d \theta
$$

The average symbol-error probability $P_{e}^{1 T_{x}-4 R_{x}}$ can be determined by evaluating the integral in Eq. (38), where $p_{\varepsilon_{1}}\left(\varepsilon_{1}\right)$ is given by Eq.(37). From Eq. (40) we can write for

$$
\beta=\frac{\pi}{2}, \quad P_{\substack{e, \beta=\frac{\pi}{2} \\ 1 T_{x}-4 R_{x}}}=\left[\frac{1}{2}-\frac{\left(\frac{35 c}{16}+\frac{35 c^{2}}{8}+\frac{7}{2} c^{3}+c^{4}\right)}{2(c+1)^{3}} \frac{1}{\sqrt{c(c+1)}}\right]
$$

and for $\beta=\frac{\pi}{4}$ 


$$
P_{e, \beta=\frac{\pi}{4}}^{3 T_{x}-1 R_{x}}=\frac{1}{\pi}\left[\frac{\pi}{4}+\frac{1}{(c+1)^{3}}\left[\begin{array}{l}
\left\{\frac{\left(\frac{29}{16} c+\frac{13}{8} c^{2}+\frac{1}{8} c^{3}\right)}{(2 c+1)}\right\}+\frac{c^{3}}{6(2 c+1)^{3}} \\
+\left\{\frac{\left(-\frac{19}{24} c^{2}-\frac{1}{4} c^{3}\right)}{12(2 c+1)^{2}}\right\}+\left(-\frac{35 c}{16}-\frac{35 c^{2}}{8}-\frac{7}{2} c^{3}-c^{4}\right) \\
\left\{\frac{1}{\sqrt{c(c+2)}} \tan ^{-1}\left(\sqrt{\frac{c+2}{c}}\right)\right\}
\end{array}\right]\right]
$$

Using Eq. (42) and Eq. (43) the average symbol-error probability for M-QAM modulation can be given as

$$
P_{e}^{1 T_{x}-4 R_{\chi}}=4 q \times P_{e, \beta=\frac{\pi}{2}}^{1 T_{x}-4 R_{x}}-4 q^{2} P_{e, \beta=\frac{\pi}{4}}^{1 T_{x}-4 R_{x}}
$$

where $c=\Delta g$ and $g=\frac{3}{2}(M-1)$

\subsection{Two transmitters-two receivers}

The PDF of instantaneous SNR per symbol $\varepsilon_{3}$ becomes

$$
P_{\varepsilon}\left(\varepsilon_{3}\right)=\frac{8 \varepsilon_{3}^{3}}{3 \Delta^{4}} \exp \left(\frac{-2 \varepsilon_{3}}{\Delta}\right)
$$

The average probability of error $P_{e}^{2 T_{x}-2 R_{x}}$ for the system can be calculate as

$$
P_{e}^{2 T_{x}-2 R_{x}}=\int_{0}^{\infty} P_{e}\left(\varepsilon_{3}\right) p_{\varepsilon}\left(\varepsilon_{3}\right) d \varepsilon_{3}
$$

\subsubsection{M-ary PSK modulation}

The symbol-error probability $P_{e}\left(\varepsilon_{3}\right)$ for M-ary PSK modulation can be given as

$$
P_{e}\left(\varepsilon_{3}\right)=\frac{1}{\pi} \int_{0}^{\beta} \exp \left(\frac{-\varepsilon_{3} a}{\sin ^{2} \theta}\right) d \theta
$$

Substitution of (45) and (47) in (46) and performing the integral [13] the equation becomes 


$$
P_{e}^{2 T_{x}-2 R_{x}}=\frac{1}{\pi}\left[\beta+\frac{1}{\left(b_{1}+2\right)^{3}}\left[\begin{array}{l}
\left\{\frac{\left(29 b_{1}+13 b_{1}^{2}+2 b^{3}\right) \sin \theta \cos \theta}{2\left(b_{1}+2 \sin ^{2} \theta\right)}\right\}+\frac{4 b_{1}^{3} \tan \theta \cos ^{6} \theta}{3\left(b_{1}+2 \sin ^{2} \theta\right)^{3}} \\
+\left\{\frac{\left(-19 b_{1}^{2}-3 b_{1}^{3}\right) \tan \theta \cos ^{4} \theta}{3\left(b_{1}+2 \sin ^{2} \theta\right)^{2}}\right\}+\left(-\frac{35 b_{1}}{2}-\frac{35 b_{1}^{2}}{2}-7 b_{1}^{3}-b_{1}^{4}\right) \\
\left\{\frac{1}{\sqrt{b_{1}\left(b_{1}+2\right)}} \tan ^{-1}\left(\sqrt{\frac{b_{1}+2}{b_{1}}} \tan \theta\right)\right\}
\end{array}\right]\right.
$$

\subsection{2. $M-Q A M$ modulation}

The symbol-error probability $P_{e}\left(\varepsilon_{3}\right)$ for square M-QAM modulation can be given as

$$
P_{e}\left(\varepsilon_{3}\right)=\frac{4 q}{\pi} \int_{0}^{\pi / 2} \exp \left(\frac{-\varepsilon_{3} g}{\sin ^{2} \theta}\right) d \theta-\frac{4 q^{2}}{\pi} \int_{0}^{\pi / 4} \exp \left(\frac{-\varepsilon_{3} g}{\sin ^{2} \theta}\right) d \theta
$$

The average symbol-error probability $P_{e}^{2 T_{x}-2 R_{x}}$ can be determined by evaluating the integral in Eq. (46), where $p_{\varepsilon}\left(\varepsilon_{3}\right)$ is given by Eq. (47). From Eq. (48) we can write for

$$
\beta=\frac{\pi}{2}, \quad P_{e, \beta=\frac{\pi}{2}}^{2 T_{x}-2 R_{x}}=\left[\frac{1}{2}-\frac{\left(\frac{35 b_{1}}{2}+\frac{35 b_{1}^{2}}{2}+7 b_{1}^{3}+b_{1}^{4}\right)}{2\left(b_{1}+2\right)^{3}} \frac{1}{\sqrt{b_{1}\left(b_{1}+2\right)}}\right]
$$

and for $\beta=\frac{\pi}{4}$

$$
P_{e, \beta=\frac{\pi}{4}}^{2 T_{x}-2 R_{x}}=\frac{1}{\pi}\left[\frac{\pi}{4}+\frac{1}{(c+2)^{3}}\left[\begin{array}{l}
\left\{\frac{\left(29 b_{1}+13 b_{1}^{2}+2 b^{3}\right)}{4(c+1)}\right\}+\frac{c^{3}}{6(c+1)^{3}} \\
+\left\{\frac{\left(-19 b_{1}^{2}-3 b_{1}^{3}\right)}{12(c+1)^{2}}\right\}+\left(-\frac{35 c}{2}-\frac{35 c}{2}-7 c-c\right) \\
\left\{\frac{1}{\sqrt{c(c+2)}} \tan ^{-1}\left(\sqrt{\frac{c+2}{c}}\right)\right\}
\end{array}\right]\right.
$$

Using Eq. (49) and Eq. (50) the average symbol-error probability for M-QAM modulation can be written as

$$
P_{e}^{2 T_{x}-2 R_{x}}=4 q \times P_{e, \beta=\frac{\pi}{2}}^{2 T_{x}-2 R_{x}}-4 q^{2} P_{e, \beta=\frac{\pi}{4}}^{2 T_{x}-2 R_{x}}
$$

\section{Performance Results}

Following the analytical approach we compute the symbol error rate performance of wireless communication system using Alamouti scheme and MRRC scheme. The symbol 


\section{Symbol Error Performance}

error rates using two transmitter-one receiver antenna and two transmitter-two receiver antenna for BPSK and QPSK modulation depicted in Figs. 1 and 2, respectively.

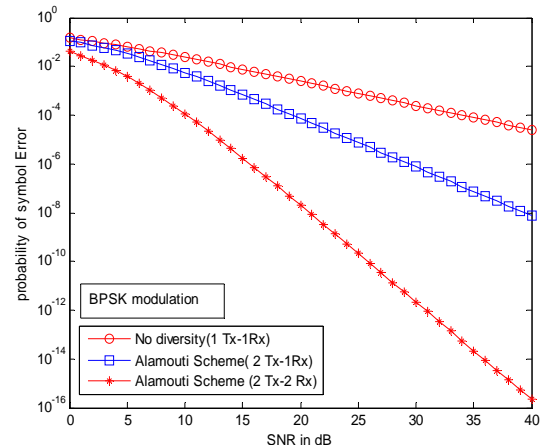

Fig. 1. Symbol Error probability of Alamouti scheme (2Tx-1Rx) and (2Tx-2Rx) for BPSK modulation in Rayleigh fading. (Analytical Derivation)

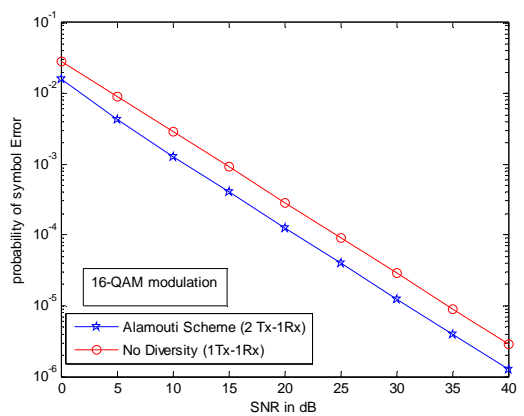

Fig. 3. Symbol error probability of Alamouti scheme $(1 \mathrm{Tx}-1 \mathrm{Rx})$ and $(2 \mathrm{Tx}-1 \mathrm{Rx})$ for 16-QAM modulation in Ravleigh fading (analvtical derivation).

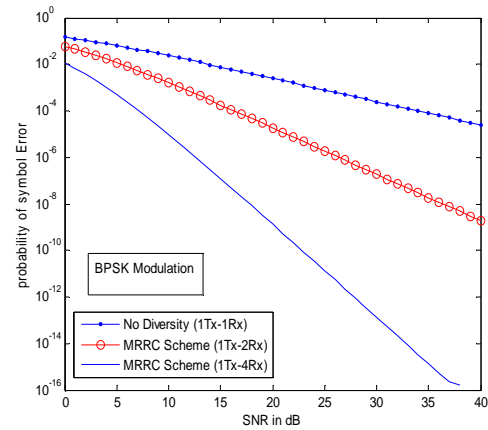

Fig. 5. Symbol error probability of MRRC scheme $(1 \mathrm{Tx}-2 \mathrm{Rx})$ and $(1 \mathrm{Tx}-4 \mathrm{Rx})$ for BPSK modulation in Rayleigh fading (Analytical derivation).

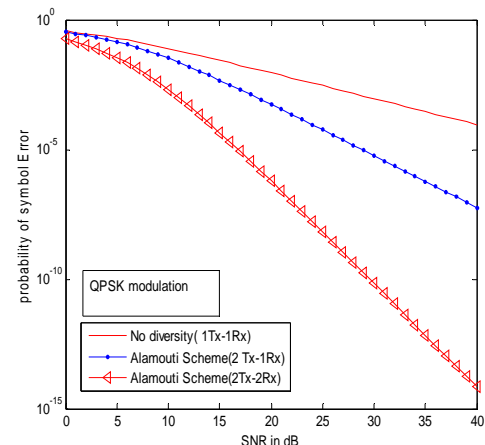

Fig. 2. Symbol Error probability of Alamouti Scheme (2Tx-1Rx) and (2Tx-2Rx) for QPSK modulation in Rayleigh fading (Analytical derivation).

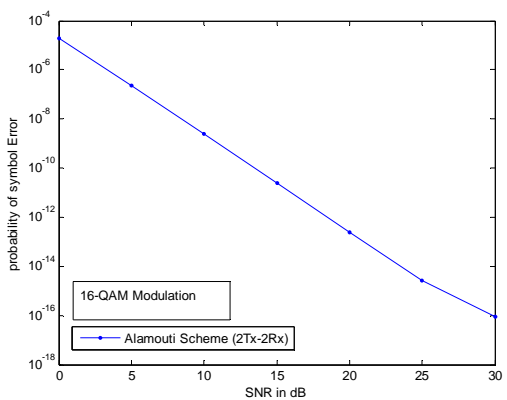

Fig. 4. Symbol error probability of Alamouti scheme (2Tx-2Rx) for 16-QAM modulation in Rayleigh fading (analytical derivation).

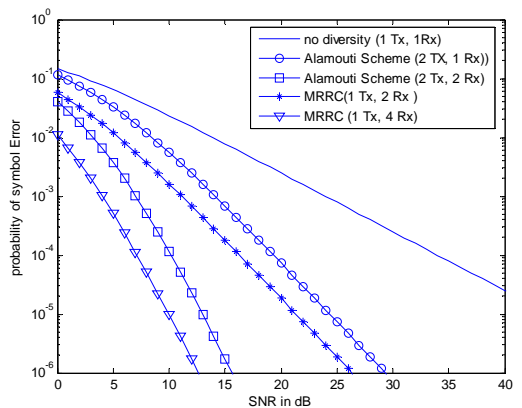

Fig. 6. Comparison of symbol error performance between maximum ratio receive combining scheme (MRRC )and Alamouti-STBC scheme for BPSK modulation in Ravleigh fading (analvtical derivation). 
It is evident from Fig. 1 and Fig. 2 that significant improvement of the SEP performance is achieved by applying diversity scheme and as the number of transmitting antenna increases probability of error decreases. The symbol error probability of Alamouti scheme using one and two transmit antenna with single receive antenna for 16-QAM modulation in Rayleigh fading is shown in Fig. 3. Fig. 4 shows the symbol error probability for two transmitter- two receiver for 16-QAM modulation. The symbol error probability of MRRC scheme using one transmitter-two receiver and one transmitter-four receiving antenna for BPSK modulation in Rayleigh fading shown in Fig. 5. The comparison between Alamouti scheme and MRRC scheme is shown in Fig. 6. The maximum ratio receive combining scheme (MRRC) technique that was used for Fig. 6 employed one transmit antenna and two receive antennas. It is evident from the Fig. 6 that adding another transmit-antenna and employing temporal diversity leads to a significant boost in symbol error performance. The Alamouti scheme gradually outperforms the MRRC technique as the SNR values increase. The MRRC technique outperforms the Alamouti scheme with just one receive antenna. The graph shows about a 3-dB penalty in error rate performance. Alamouti explains this penalty by noting a constraint that he imposes on his code; namely, he has each transmit antenna radiating half of the energy that the one antenna for the MRRC scheme radiates. The Alamouti scheme is practical because it allows a boost in performance through the addition of receiver antennas. The Monte Carlo simulation of symbol error rate performance of Alamouti coded system for BPSK modulation in Rayleigh fading for 2Tx-1Rx and 2Tx2Rx are depicted in Figs. 7 and 8, respectively. It is seen form these figures that, Monte Carlo simulation showed an exact match with the theoretical results.

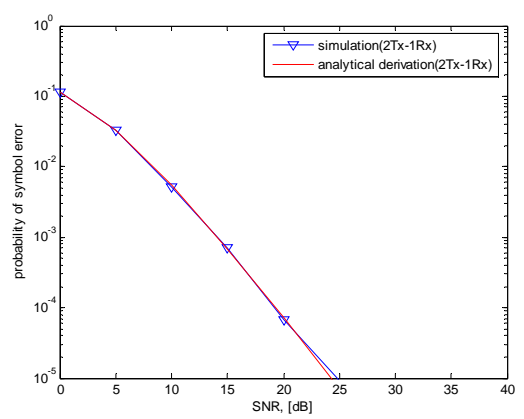

Fig. 7. Symbol error performance comparison of Alamouti scheme(2Tx-1Rx), monte carlo simulation result and analytical derivation for BPSK modulation in Rayleigh fading

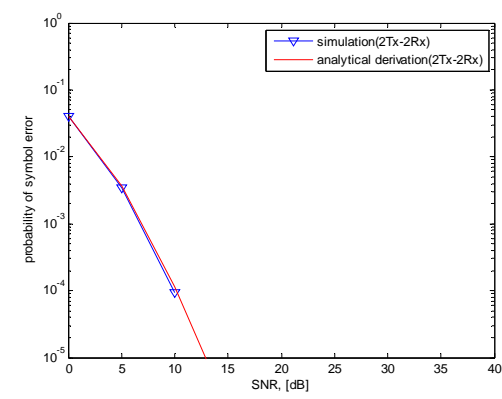

Fig. 8. Symbol error performance comparison of Alamouti scheme(2TX-2Rx), monte carlo simulation result and analytical derivation for BPSK modulation in Rayleigh fading

\section{Conclusion}

We investigate the probability of error of single-input-single-output (SISO) system and Alamouti scheme using 2 Tx-1Rx, 2Tx-2Rx and MRRC scheme using 1Tx-2Rx, 1Tx-4Rx 
in Rayleigh fading channels. A detailed analysis for finding the symbol error probability of the wireless communication systems in Rayleigh fading channels is provided. Exact closed-form expressions are derived for average symbol-error-probability (SEP) for both M-PSK and M-QAM modulation schemes. The numerical results show exact match with Monte Carlo simulation.

\section{References}

1. J. G. Proakis and M. Salehi, Communication System Engineering, $2^{\text {nd }}$ Edition (Third Indian Reprint, Pearson Education, 2004).

2. K. Feher, Wireless Digital communication (Modulation and spread Spectrum Application), (Prentice-Hall, 2005) pp 335-352.

3. L. Hanzo, M. Munster, B. J. Choi, and T. Keller, OFDM and MC-CDMA for Broadband Multiuser Communications, WLANs and Broadcasting (Reprinted March 2004, June 2004).

4. V. K. Grag, Wireless Communication and Networking (Morgan Kaufmann Publishers, 2007).

5. WIKIPEDIA, the free Encyclopedia - Microsoft Internet Explorer and Websites based on MIMO-OFDM with STBC, Different Multiplexing and Multiple accesses, WiMAX, Bluetooth, Diversity and different codes.

6. S. M. Alamouti, IEEE J. Selected Areas in Communications 1 (8), 1451 (1998). doi:10.1109/49.730453

7. V. Tarokh, H. Jafarkhani, and A. R. Calderbank, IEEE Trans. Inform. Theory 45 (5), 1456 (July 1999). doi:10.1109/18.771146

8. V. Tarokh, H Jafarkhani, and A. R. Calderbank, IEEE J. Selected Areas in Communications 17 (3), 451 (March, 1999). doi:10.1109/49.753730

9. X. Li, T. Luo, G Yue, and C Yi, IEEE Trans. Communication 49 (10),1700 (October, 2001). doi:10.1109/26.957388

10. C. R. N. Athaudage and K. Sathananthan, Proc. IEEE International Conference on Communications 4, 2593 (ICC’05), (Seoul, Korea, May 2005). doi:10.1109/ICC.2005.1494818

11. J. G. Proakis, Digital Communications, $4^{\text {th }}$ Edition (McGraw-Hill Series, 2001).

12. M. K. Simon and M. S. Alouini, Digital Communication Over Fading Channels: A Unified Approach to Performance Analysis (Wiley, New York, 2000). doi:10.1002/0471200697

13. I. S. Gradshteyn and I. M. Ryzhik, Table of Integrals, Series, and Products, $6^{\text {th }}$ ed. (Academic Press Inc., 2001). 